Background Previous research has shown that muscle imbalances may be an important risk factor for lower limb injuries in runners. No studies have evaluated hip musculature imbalance in sprinters. Identifying pre-season strength and imbalances of the hip gluteal musculature can have an important impact on injury prevention within this population.

Objective To describe the muscle performance and imbalances between limbs related to the hip extensor, external rotators and abductors muscles in sprinters.

Design Athletes were assessed during the pre-season period. Muscle function performance of the hip extensors, external rotators and abductors were tested.

Participants All participants $(n=69)$ were federated at the Spanish athletics federation and involved in high-level track and field training sessions (minimum 5 sessions per week) for at least the last 3 years. All of them had undertaken similar training demands in the past.

Interventions We measured the maximum number of repetitions without compensatory movements of each leg during the straight knee bridge exercise (test 1), hip external rotation in prone (test 2) and hip abduction in side-line (test 3). Data analysis and comparison in between limbs were calculated.

Results No significant differences in between limbs were observed in all three tests $(p<0,005)$. The assessment of the hip external rotators (test 2), have shown the worse performance among this population with $30,8 \%$ of the athletes on the dominant limb and $35,3 \%$ on non-dominant limb not being able to perform even 1 rep without compensation.

Conclusions We did not observe significant imbalances in between limbs. The hip external rotators muscles demonstrated the worst performance with a high number of athletes not being able to perform the test. The weakness of the external rotators and compensations associated with it can have several different impacts on the lower limb function during running, therefore possible importance for injury prevention strategies.

\section{ISOKINETIC PROFILE AND CONTRALATERAL DEFICIT OF THE LOWER LIMBS OF ARTISTIC GYMNASTICS ATHLETES}

\footnotetext{
1,2Stefânia Gonçalves Dias, ${ }^{2}$ Natália Batista Albuquerque Goulart Lemos, ${ }^{3}$ Morgana Lunardi, ${ }^{2}$ Fernando de Aguiar Lemos, ${ }^{4}$ Caroline Pieta Dias, ${ }^{4}$ João Carlos Oliva, ${ }^{4}$ Marco Aurélio Vaz. ${ }^{1}$ University of Pernambuco, Petrolina, Brazil; ${ }^{2}$ Federal University of Vale do São Francisco, Petrolina, Brazil; ${ }^{3}$ Federal University of Santa Catarina, Florianópolis, Brazil; ${ }^{4}$ Federal University of Rio Grande do Sul, Porto Alegre, Brazil
}

10.1136/bjsports-2021-IOC.234

Background The hamstring/quadriceps (H/Q) functional ratio detects muscle imbalance and monitors knee joint stability, making it a predictor of injury, especially ACL. The injury of this ligament in gymnasts is one of the most incident and of greatest negative impact on recovery and return to sports.

Objective To evaluate functional $\mathrm{H} / \mathrm{Q}$ at different angular velocities and contralateral deficit of the lower limbs of female artistic gymnastics athletes.

Design Descriptive study.

Setting Federated athletes, participants of competitions at national and international level, in which two were part of the Brazilian Selection of Artistic Gymnastics.

Patients (or Participants) Selected through a club in Porto Alegre, Brazil. They had an average of eight years of experience in the modality, minimum frequency of training of six times a week, six hours daily. Participants with any lesion that interfered or made it impossible to perform the evaluations were excluded.

Interventions (or Assessment of Risk Factors) Functional H/Q was calculated by dividing the peak of the hamstring eccentric torque by the peak of the quadriceps concentric torque, analyzed using the isokinetic dynamometer (Biodex Medical System).

Main Outcome Measurements Isokinetic protocol at two speeds: 1) $60 \%$ s and 2) $120 \%$ s; and Waterloo questionnaire to determine dominant member (DM).

Results 10 elite gymnasts were evaluated, (mean \pm SD age 14.9 \pm 3.7 yrs; body mass $45.6 \pm 7.0 \mathrm{~kg}$ and height $1.51 \pm 0.04 \mathrm{~m}$ ). The DM, as well as the non-dominant (NDM) at the moment $60 \%$ s $(0.71 \pm 0.09 ; 0.77 \pm 0.12)$, had values below those established in the literature (functional $\mathrm{H} / \mathrm{Q} \leq 0.90$ ), at the moment $120^{\circ} \mathrm{s}$ were closer to normal $(0.85 \pm 0.12 ; 0.88 \pm$ 0.13). 5 of the 10 athletes obtained values within the normal range in the MND at $120 \%$ s.

Conclusions All athletes presented significant thigh muscle imbalance, with functional $\mathrm{H} / \mathrm{Q}$ values lower than those proposed in the literature, with proximity between the limbs. Strength training program of hamstrings should be considered in order to prevent injuries caused by muscle imbalance.

\section{THE DIFFERENCES OF STATIC LOWER EXTREMITY ALIGNMENT BETWEEN FEMALE BALLET STUDENTS AND FEMALE NON-BALLET STUDENTS}

${ }^{1,2}$ Min Jin Kim, ${ }^{1,2,3}$ Sae Yong Lee. 'Department of Physical Education, Yonsei University, Seoul, South Korea (Republic of); ${ }^{2}$ YISSEM (Yonsei Institute of Sports Science and Exercise Medicine), Seoul, South Korea (Republic of); ${ }^{3}$ Institute of Convergence Science, Seoul, South Korea (Republic of)

\subsection{6/bjsports-2021-IOC.235}

Background Ballet dancers experience changes in body alignment in repeated practice to achieve the ideal turn-out position, and they have different alignments from that of the nonballet dancers. However, no quantitative data have been reported to support their differences in lower extremity alignment (LEA).

Objective To compare differences in clinical measures of static LEA between female ballet students and female non-ballet students.

Design Descriptive and cohort study.

Setting Controlled laboratory setting.

Patients (or Participants) Eighty-four female ballet students and fifty-one female non-ballet students were recruited.

Interventions (or Assessment of Risk Factors)

Every participant read and signed the concert form that approved by the Institutional Reviews Board (IRB). The static LEAs were measured using four instruments, Height gage, 6' and 12' Goniometers, PALM inclinometer, and Bubble inclinometer.

Main Outcome Measurements Twelve static LEAs includeing leg-length, quadriceps angle, tibiofemoral angle, prone rearfoot angle, forefoot angle, tibial torsion, tibial varum, hip anteversion, pelvic tilt, standing quadriceps angle, standing rearfoot angle and navicular drop were measured.

Results Ballet students showed greater mean in tibial torsion $\left(\mathrm{M}_{\text {diff }}=4.55, S E=.83, t_{(266)}=5.51, p=.000,95 \%\right.$ CI: $2.92-$ $6.18)$, anterior-posterior pelvic tilt $\left(\mathrm{M}_{\text {diff }}=1.93, S E=.46, t_{(228)}\right.$ $=4.10, p=.000,95 \% \mathrm{CI}: 1.00-2.86)$, and standing rearfoot 
angle $\quad\left(\mathrm{M}_{\text {diff }}=4.81, S E=.51, t_{(268)}=9.42, p=.000,95 \% \mathrm{CI}:\right.$ 3.81 - 5.82) than non-ballet students. In contrast, ballet students had lesser mean than non-ballet students in tibiofemoral angle tibiofemoral angle $\left(\mathrm{M}_{\text {diff }}=-1.05, S E=.31, t_{(268)=-3.39}\right.$, $p=.001,95 \%$ CI: $-1.64 \quad-0.44)$, prone rearfoot angle $\left(\mathrm{M}_{\text {diff }}=-8.65, S E=.56, t_{(254.04)=}-16.16, p=.000,95 \% \mathrm{CI}:-9.71\right.$ - -7.60), tibial varum $\left(\mathrm{M}_{\text {diff }}=-2.52, S E=.23, t_{(159.90)}=-10.98\right.$, $p=.000$, 95\% CI: $-2.96--2.06)$, hip anteversion $\left(\mathrm{M}_{\text {diff }}=-\right.$ 11.47, $S E=.72, t_{(156.15)}=-15.87, p=.000,95 \%$ CI: $-12.90--$ $10.04)$, and navicular drop $\left(\mathrm{M}_{\text {diff }}=-4.45, S E=.42, t_{(182.22)=^{-}}\right.$ 10.44, $p=.000,95 \%$ CI: $-5.29--3.61)$.

Conclusions Significant results from the alignment indicate that ballet movement and turn-out position may suggest changes in LEA, therefore, clinicians should consider these aspects while preventing and treating dancer injuries.

\section{INFLUENCE OF LOWER QUARTER Y-BALANCE TESTTM SCREENING PROTOCOL ON DYNAMIC BALANCE OUTCOMES}

${ }^{1}$ Scott Lawrance, ${ }^{1}$ Megan Jacobs, ${ }^{1}$ Emily Boss, ${ }^{1}$ Jennifer Popp, ${ }^{1}$ Larry Leverenz, ${ }^{2}$ Michael Weller. ${ }^{1}$ Purdue University, West Lafayette, IN, USA; ${ }^{2}$ Cedarville University, Cedarville, OH, USA

\subsection{6/bjsports-2021-IOC.236}

Background The Lower Quarter Y-Balance Test ${ }^{\mathrm{TM}}$ (LQYBT) is commonly used to determine risk of injury or readiness for return to sport. However, clinicians conduct the assessment with differing test protocols potentially altering test outcomes. Objective To determine if differences in reach distances, composite score, and limb symmetry exist between LQYBT testing protocols.

Design Prospective cohort study.

Setting Controlled laboratory research.

Participants 48 non-injured participants: 32 females, 16 males $(21.4 \pm 0.3$ years, $170.6 \pm 9.2 \mathrm{~cm}, 72.9 \pm 14.2 \mathrm{~kg})$.

Interventions Participants completed four testing protocols including barefoot with hands fixed at their hips, barefoot with hands free to move, shod with hands fixed at hips, and shod with hands free to move.

Main Outcome Measures Maximum reach distance was recorded for each limb in the anterior, posteromedial, and posterolateral directions. Limb length composite scores and limb symmetry index (LSI) were calculated for each LQYBT assessment.

Results No statistically significant differences were observed in reach distances, composite scores, or LSI between shod and barefoot protocols. Significant differences were observed in reach distances $(p=0.00)$ and in limb length composite scores (right limb $p=0.00$, left limb $p=0.00$ ) between protocols comparing hands fixed at hips and hand free to move, although no differences were observed in LSI between these conditions when participants were shod $(p=0.27)$ or barefoot $(p=0.49)$.

Conclusions No differences were observed when participants wore athletic shoes or were barefoot during assessments. Reach distance and limb composite score differences were present when participants were allowed to move their arms and counterbalance their movement during the LQYBT, although no differences in LSI was observed. Results suggest if LSI is used to make clinical decisions, any LQYBT testing protocol can be used. However, testing protocols could influence clinical decisions if reach distances or composite scores are used to make patient care decisions.

\section{8 SPORT PRE-PARTICIPATION HEALTH EVALUATION IN ELITE ATHLETES FROM A MULTISPORT CLUB: PROPOSAL FOR A PERSONALIZED PROTOCOL}

${ }^{1}$ Ramon Pi, 1,2María Sanz de la Garza, ${ }^{3}$ Gonzalo Grazioli, 1,2Gil Rodas, 'Manel García, ${ }^{1,2}$ Marta Sitges, ${ }^{1,4}$ Francheck Drobnic. ${ }^{1}$ FC Barcelona Medical Department, Barcelona, Spain; ${ }^{2}$ Hospital Clínic, Barcelona, Spain; ${ }^{3}$ Sports Medicine Unit, Consell Catalá de l'Esport, Barcelona, Spain; ${ }^{4}$ Olympic Training Centre, Sant Cugat del Valles, Barcelona, Spain

\subsection{6/bjsports-2021-IOC.237}

Background In 2009 the IOC published a Consensus Statement highlighting the value of periodic health evaluation in elite athletes.

Objective The aim of this study was to evaluate the prevalence of pathological findings, and the preventive value of the method used.

Design Retrospective epidemiological study.

Setting and Participants A total of 2574 elite and professional athletes from Football Club Barcelona (FCB). FCB is a sports club with 13 different sports, 5 professional and 8 amateur. Interventions (or Assessment of Risk Factors) Between 2008 and 2018, a total of 2574 athletes, with range from 12 to 35 years old, were evaluated through a personalized sport preparticipation health evaluation protocol (SPPHE) at the FCB Medical Department.

Main Outcome Measurements The protocol used was the 'Guía de la Revisión Médica del Futbol Club Barcelona'. The SPPHE examination consisted of basic medical information, anthropometric data, physical examination, spirometry, basal 12-lead electrocardiography, submaximal cardiovascular exercise testing, and cardiac echocardiography.

Results In 2574 SPPHE, we recorded 750 medical findings (29.1\%); including 495 (19.2\%) pathological findings, 255 (9.9\%) minor abnormal findings, and 958 (37.2\%) previous sports injuries. Specific treatment was necessary in 6 cardiovascular diseases. We found 3 endocrinological diseases (type 1e Diabetes Mellitus). Periodic follow-up was specified in all cases, although none prevented the athlete from competing.

Conclusions The SPPHE was effective in identifying a wide range of pathologies $(66.3 \%)$ in elite athletes from this multisport club. This allow us to provide fast treatment and implement ad hoc strategic preventive programs. We found no alteration that affected the athlete's development or hindered them in reaching the highest level of competition.

\section{SHOULD THE SIDE-HOP TEST BE REDUCED FROM 40 CM TO 30 CM WHEN USED IN DEVELOPMENTAL ATHLETES?}

${ }^{1,2}$ Isabel Schneider, ${ }^{3}$ Nicolai Kammann, ${ }^{1,3}$ Oliver Haertel. ' German University of Health and Sport, Berlin, Germany; '2Physio-Motion, Groebenzell, Germany; ${ }^{3}$ Leichtathletik Foerderzentrum, Munich, Germany

\subsection{6/bjsports-2021-IOC.238}

Background The side-hop test by Gustavsson et al. (2006) helps with return to play decision making after lower extremity injury.

Objective Determine whether a reduced width of $30 \mathrm{~cm}$ (compared with $40 \mathrm{~cm}$ ) may be more suitable for use in developmental athletes.

Design Cross-sectional study.

Setting School gymnasium. 